\title{
Evaluaciones Económicas en Salud I: ¿Son válidos los resultados de este
}

estudio? (0 de porqué es a veces importante evaluar los resultados de las decisiones tanto en términos de salud como monetarios).

\section{Resumen}

En esta primera entrega de "Cómo leer un artículo" nos dedicaremos a las evaluaciones económicas de la salud. Se definen a las evaluaciones económicas en general y a los tipos principales utilizados en el área de la salud (análisis de costo-minimización, análisis de costo-beneficio, análisis de costo-efectividad y análisis de costo-utilidad). Se evalúan los criterios de validez, se explica cómo se interpretan los resultados de una evaluación económica, y si estos son aplicables a la situación particular del lector.

\section{Escenario clínico}

Usted es un médico general que trabaja en el área preventiva de su hospital de referencia. El director del hospital, luego de leer los resultados que mostraban la reducción de la mortalidad por cáncer de colon a través del rastreo del mismo, y las muy frescas recomendaciones de la fuerza de tareas de EE.UU aun no publicadas en EVIDENCIA lo llama a una reunión. La idea es discutir el caso del rastreo de cáncer colorrectal junto a un equipo de un cirujano colorrectal, un gastroenterólogo (ambos a favor de la colonoscopía), un radiólogo (que recomienda fuertemente el colon por enema), y un bioquímico (quien está a favor de la detección de sangre oculta en materia fecal por medio del guayaco). El director les dice que para la siguiente semana debe haber una recomendación hospitalaria de si debe o no recomendarse el rastreo y a través de qué prueba o pruebas."En estos tiempos de crisis es cuando más debemos ser racionales y adoptar una estrategia lo más beneficiosa posible al mejor costo posible. Recuerden que los recursos que asignemos a este programa ya no estarán disponibles para otros..."

\section{La búsqueda}

Cómo usted está buscando evaluaciones económicas en salud y tiene poco tiempo disponible, comienza por una búsqueda "específica": en el sitio del Center for Reviews And Dissemination de la Universidad de York, Inglaterra, mantienen una excelente base de datos de evaluaciones económicas con comentarios (http://agatha.york.ac.uk/nhsdhp.htm). El resultado obtenido con las palabras claves "colorectal cancer" AND "sceening" arroja 56 posibles artículos (si prueba las mismas palabras claves en PubMed le arroja la friolera de 36.679 citas, las que se reducen a sólo 347 si le agrega el término MeSH " cost-benefit analysis". Los artículos más atractivos por sus títulos y resúmenes fueron:

- Cost-effectiveness of colorectal cancer screening. McMahon $P$ M, Bosch J L, Gleason S, Halpern E F, Lester J S, Gazelle G S. Radiology 2001; 219(1):44-50.

Es una revisión basada en algunos estudios de la década de los 90', se lo elimina al no ser una evaluación original (y por no estar disponible en la biblioteca de su hospital...).

- Cost-effectiveness of a single colonoscopy in screening for colorectal cancer.Sonnenberg A, Delco F. Arch Intern Med 2002 Jan 28;162(2):163-8

Este lo descarta leyendo el abstract ya que evalúa solamente la colonoscopía (una única vez o más) y a usted le interesa estudios que comparen las alternativas principales.
- Cost-effectiveness of screening for colorectal cancer in the general population. Frazier A L, Colditz G A, Fuchs C S, Kuntz K M. JAMA 2000; 284(15): 1954-1961.

- Cost-effectiveness of colonoscopy in screening for colorectal can cer. Sonnenberg A, Delco F, Inadomi J M. Annals of Internal Medicine 2000; 133(8):647-649.

Selecciona estos últimos dos para evaluarlos en más detalle ya que le parecen pertinentes a primera vista.

La mayoría de los profesionales de la salud dedican la mayor parte de su tiempo a tareas asistenciales, a acompañar a los pacientes a tomar decisiones de cualquier tipo (¿me hago el test de la próstata para evitar el cáncer?, ¿hay que lograr una TA $<135 / 85$ en este paciente diabético?). Pero los profesionales de la salud también se involucran en la toma de decisiones sobre grupos de individuos o poblaciones (en el caso de nuestro médico una decisión acerca de una norma institucional). Estas decisiones son frecuentes tanto en el sector público hospitalario, a nivel gubernamental (por ejemplo ministerios de salud provincial o nacional al decidir la política de vacunación, o si debe tener tomógrafos en todos los hospitales públicos) y también en instituciones privadas (por ej.cuál es manejo más costo-efectivo en los pacientes diabéticos de esta prepaga?).

En estas decisiones poblacionales, debemos tener en cuenta no solo los beneficios y los riesgos de los tratamientos, sino también si los beneficios "valen la pena" en relación a la cantidad de recursos que consumen. En nuestros países, donde los recursos en salud son más limitados, es fundamental invertir los recursos de la mejor manera posible (con el presupuesto que hay, cuál es la mejor manera de "comprar" más salud).

Es en estas situaciones donde las evaluaciones económicas de salud de las prácticas clínicas nos pueden ayudar. Las evaluaciones económicas son un grupo de métodos formales y cuantitativos utilizados para comparar estrategias alternativas en relación tanto a los resultados en la salud como a los recursos y costos insumidos. Es una herramienta que intenta informar decisiones de asignación de recursos, ayudan a tomar decisiones (aunque el que toma las decisiones puede hacer lo que le plazca finalmente...). Son estudios cada vez más frecuentes en publicaciones de salud. Los datos acerca de la eficacia de los tratamientos son en general provistos por ensayos clínicos aleatorizados o revisiones sistemáticas de los mismos.A veces también recolectan datos de utilización de recursos o costos de tratamientos. Las evaluaciones económicas, así como otros estudios integradores como las guías de práctica clínica o los análisis de decisión, utilizan estimaciones de efectividad y de costos tomados de la literatura. La principal diferencia de las evaluaciones económicas con el resto es la medición explícita y la valuación de los recursos consumidos o los costos. La integración de los datos de costos implica muchas veces integrar y valorar también los resultados sanitarios de un modo que puedan ser comparados con estrategias de tratamiento alter nativas.

Para poder entender esto, que a veces parece muy ajeno a la práctica cotidiana y sin embargo es de suma utilidad para elegir bien las cosas a hacer, haremos una revisión de cómo estos estudios son realizados así como sus puntos fuertes y débiles. Los más curiosos pueden ir a algunos de los artículos o libros seminales en el tema1-7.

Como es costumbre, intentaremos aproximarnos al análisis de artículos de evaluaciones económicas de estrategias clínicas con las tres preguntas básicas (tabla 1): 
Tabla 1. Preguntas para leer una evaluación económica

\section{1. ¿Son los resultados del estudio Válidos?}

Esta pregunta se refiere a si la evaluación económica determina certeramente cuál de las estrategias clínicas provee el mayor beneficio con los recursos disponibles. Así como con otro tipo de estudios, la validez de una evaluación económica está básicamente determinada por su calidad metodológica.

\section{2.¿Cuáles fueron los resultados?}

Si la respuesta a la primer pregunta fue afirmativa, y la evaluación provee una estimación sin sesgos de los costos y las consecuencias de las distintas estrategias evaluadas, sigamos adelante y evaluemos los resultados. Aquí deberemos evaluar la magnitud de los beneficios esperados y los costos al adoptar la estrategia más eficiente, así como el nivel de incertidumbre que los rodea

3. ¿Ayudarán los resultados al cuidado de mis pacientes?

Si los resultados son válidos e importantes, el próximo paso es examinar cómo aplicarlos en su contexto clínico o sanitario.

\section{Tabla 2. Guías para el lector de evaluaciones económicas}

\section{1. ¿Son los resultados del estudio Válidos?}

¿Provee el análisis una comparación económica completa de las distintas estrategias de salud?

¿Compara estrategias bien definidas?

$¿$ ¿Con una perspectiva específica para evaluar los costos y resultados?

¿Tiene evidencia válida acerca de la eficacia de las alternativas?

¿Identifica todos los costos y efectos que debería, y los mide y valora en forma adecuada?

¿Fue el tipo de análisis adecuado a la pregunta realizada? ¿Se dio cuenta de la incertidumbre en el análisis?

\section{2. ¿Cuáles fueron los resultados?}

¿Cuáles fueron los costos y los resultados incrementales de cada estrategia?, ¿Son clínicamente significativos? ¿Difieren los costos y resultados incrementales entre distintos subgrupos?

¿Cuánto cambian los resultados al incorporar la incertidumbre?

3. ¿Ayudarán los resultados al cuidado de mis pacientes?

¿Valen la pena los beneficios del tratamiento en relación a los daños y los costos?

¿Podrían mis pacientes esperar similares resultados?

¿Son los costos similares?

\section{1. ¿Son los resultados del estudio Válidos?}

¿Provee el análisis una comparación económica completa de las distintas estrategias de salud?

Las evaluaciones económicas comparan dos o más tratamientos, programas, o estrategias. Si se analizan dos estrategias solo en términos monetarios miraría la mitad del vaso (el consumo de recursos) y se denomina análisis de costo.Del mismo modo estudios de la eficacia comparativa de dos estrategias como un ensayo clínico informa solo acerca de la otra mitad, la de los resultados. Una evaluación económica completa requiere que tanto los costos como las consecuencias sean analizadas para cada una de las estrategias comparadas. Los distintos tipos de evaluaciones económicas completas se diferencian por la manera de medir los beneficios (ver tabla 3 ).

\section{Tabla 3. Diferentes formas de evaluación económica}

\begin{tabular}{l|l}
\hline Tipo de estudio & Medición de los beneficios \\
\hline Análisis de costo-minimización & Cuando los beneficios fueron equivalentes \\
Análisis de costo-efectividad & Unidades naturales (por ej. Años de vida ganados) \\
Análisis de costo-utilidad & Años saludables (por ej. QALYS [años de vida ajustados por calidad]) \\
Análisis de costo-beneficio & Se le asigna un valor monetario a los beneficios de la salud \\
\hline
\end{tabular}

¿Compara estrategias bien definidas (sin omisiones)?

Es importante que las estrategias elegidas para compararse sean las adecuadas, o sea las alternativas relevantes que uno piensa adecuadas para el problema. Incluso muchas veces es adecuado comparar las estrategias "activas", con una estrategia de seguimiento o "status quo", si es razonable.

En el caso de nuestros estudios, el de Frazier parece incluir todas las alternativas relevantes: 22 estrategias de colonoscopía, SOMF rehidratada o no, sigmoidoscopía o enema de bario de doble contraste, en distintos regímenes. En cambio el de Sonnenberg solo evalúa SOMF, sigmoidoscopía y colonoscopía.Ambos estudios incluyen la estrategia de no realizar rastreo como la alternativa contra la que el resto se compara, lo cual es razonable hoy en día que el rastreo no está muy extendido (a pesar de ser una recomendación tipo $\mathrm{B}^{\star}$ ).

Una de las limitaciones de las evaluaciones económicas es que las comparaciones se encuentran limitadas por la disponibilidad de datos de estudios clínicos.Un caso especial es que en los ensayos clínicos de muchas nuevas medicaciones la comparación es con el placebo más que con otro tratamiento activo. Esto implica que, en muchas ocasiones, las evaluaciones económicas no se basen en un único ensayo clínico sino que en la integración de varios, y esto implica utilizar una serie de asunciones. Es por ello que los usuarios de evaluaciones económicas debemos chequear los métodos de los ensayos clínicos utilizados y si estos son realmente comparables. Se debe mirar si los estudios clínicos utilizados incluían pacientes similares o no, o medían los resultados clínicos de una forma homogénea. Como no existe un ensayo clínico que compare todos los tests de rastreo de cáncer de colon a la vez, ambos estudios evaluados combinan datos de muchos estudios distintos de población general, lo parece razonable.

\section{¿Con una perspectiva específica para evaluar los costos y resultados?}

Tanto los costos como las consecuencias pueden ser analizados desde múltiples puntos de vista: el del paciente, el del hospital, el de un sistema de medicina prepaga, o el de la sociedad en su conjunto. Cada perspectiva puede ser relevante en distintas ocasiones, pero en general la perspectiva más amplia es la adecuada para la alocación de recursos globales de salud. una evaluación que adopta, por ejemplo, la perspectiva hospitalaria, puede ser útil en la asignación de recursos del presupuesto del hospital. Muchas 
veces, sin embargo, las evaluaciones económicas apuntan a informar políticas desde una perspectiva más amplia, la de toda la sociedad (como ejemplo para ilustrar las distintas perspectivas en el caso de un programa de reinserción precoz de esquizofrénicos en la sociedad, el mismo puede implicar un ahorro de costos del presupuesto del hospital por menos días de internación; sin embargo, desde la perspectiva social, los costos para los pacientes y sus familias pueden aumentar).

Pero muchas veces se considera una perspectiva menos amplia para evaluar el impacto en los principales actores presupuestarios, incluso en Argentina donde las decisiones presupuestarias son realizadas de manera variada y muchas veces decentralizada (algunas el sistema público, otras el sistema de obras sociales y de medicina prepaga, la seguridad social, etc.).

En honor a la verdad se debe decir que, aunque recomendada, las evaluaciones desde el punto de vista social son poco frecuentes. Esto se debe principalmente a que es muy difícil rastrear otros costos fuera del sistema de salud (por ejemplo el dinero usado por el paciente y la familia para los tratamientos, para transporte, el lucro cesante, el llamado cuidado informal si un familiar o cuidador necesita cuidarlo, etc.De todos modos, la perspectiva del paciente es parcialmente tenida en cuenta en el análisis al medir los resultados del tratamiento, por ejemplo el impacto en la calidad de vida.

En el caso de los dos estudios que decidió evaluar, ambos evalúan las estrategias desde la perspectiva del sector de salud solamente. En la tabla 4 Hacemos una comparación entre estos dos trabajos a evaluar en lo que respecta a algunos aspectos a destacar.

Tabla 4.Principales aspectos de los estudios comparados

\begin{tabular}{l|l|l}
\hline Característica & \multicolumn{1}{|c|}{ Frazier y cols8 } & Sonnenberg y cols9 \\
\hline Diseño global del estudio & $\begin{array}{l}\text { Análisis de costo-efectividad uti- } \\
\text { lizando un modelo Markov* }\end{array}$ & $\begin{array}{l}\text { Análisis de costo-efectividad uti- } \\
\text { lizando un modelo Markov* }\end{array}$ \\
\hline Perspectiva del análisis & "Social" ** & Financiador del sistema de salud \\
\hline Alternativas comparadas & $\begin{array}{l}\text { Colonoscopía, SOMF rehidrata- } \\
\text { da o no, sigmoidoscopía o ene- } \\
\text { ma de bario de doble contraste, } \\
\text { todos en distintos regímenes }\end{array}$ & $\begin{array}{l}\text { SOMF, sigmoidoscopía y } \\
\text { colonoscopía }\end{array}$ \\
\hline Medidas de beneficio & $\begin{array}{l}\text { Incidencia, mortalidad por cáncer } \\
\text { de colon, años de vida ganados }\end{array}$ & $\begin{array}{l}\text { Incidencia, mortalidad, años de } \\
\text { vida ganados }\end{array}$ \\
\hline $\begin{array}{l}\text { Fuente(s) de datos de } \\
\text { efectividad }\end{array}$ & $\begin{array}{l}\text { Ensayos de rastreo de cáncer de } \\
\text { colon }\end{array}$ & $\begin{array}{l}\text { Ensayos de rastreo de cáncer de } \\
\text { colon }\end{array}$ \\
\hline $\begin{array}{l}\text { Fuente(s) de datos de } \\
\text { calidad de vida }\end{array}$ & No la evalúan & No la evalúan \\
\hline $\begin{array}{l}\text { Estimación de uso de } \\
\text { recursos }\end{array}$ & $\begin{array}{l}\text { Datos epidemiológicos y del } \\
\text { modelo }\end{array}$ & Basados en DRG*** \\
\hline Fuente(s) de datos de costos & $\begin{array}{l}\text { Estudio de costos de una gran } \\
\text { prepaga de EE.UU. }\end{array}$ & $\begin{array}{l}\text { US healthcare Finance Adminis- } \\
\text { tration, y de la literatura }\end{array}$ \\
\hline Descuento & $\begin{array}{l}\text { 3\% anual } \\
\text { Influencias de la adherencia a los } \\
\text { tests, sus características opera- } \\
\text { tivas, costos, efectos adversos, } \\
\text { tasa de descuento, incidencias } \\
\text { de cáncer. }\end{array}$ & $\begin{array}{l}\text { Influencia de costos de cada } \\
\text { estrategia, frecuencia de tests, } \\
\text { características operativas, } \\
\text { adherencia }\end{array}$ \\
\hline
\end{tabular}

SOMF:Sangre oculta en materia fecal

${ }^{* *}$ aunque el estudio reporta la perspectiva de la sociedad, solo evalúan los costos acarreados por el sistema de salud

***DRGs, diagnostic related groups, es una manera de calcular los preciospagos según patología o procedimiento.

\section{¿Tiene evidencia válida acerca de la eficacia de las alternativas?}

Obviamente, la validez de una evaluación económica se sustenta en la validez de sus partes, y una esencial es la de la efectividad de las alternativas comparadas. Hay distintos tipos de evaluaciones económicas: unas se realizan en forma concurrente a un ensayo clínico controlado (Ilamadas "a caballito" o piggyback en inglés); otras se basan en una revisión sistemática de ensayos clínicos;y otras en una síntesis más amplia a través de la realización de modelos, como es el caso de nuestros estudios analizados.Aunque las que se basan en un ensayo clínico son más rigurosas y con mayor validez interna, muchas veces tienen menor validez externa ya que las prácticas dentro de un ensayo clínico en general no reflejan la práctica habitual (mayor adherencia, mejor seguimiento, etc.).

Un problema frecuente es la necesidad de tomar decisiones de importancia a largo plazo pero teniendo datos solamente de ensayos clínicos a corto o mediano término. Por ejemplo, en los estudios de estrategias hipocolesterolemiantes, datos de resultados finales como mortalidad por cualquier causa, llevan muchos años de obtener.En este caso se utilizaron modelos que realizan proyecciones a largo plazo basándose en resultados intermedios, como por ejemplo el porcentaje de descenso del colesterol LDL. La utilidad de esta aproximación depende de la fuerza de la asociación del resultado intermedio (reducción del colesterol) con el final al largo plazo (mortalidad). De todos modos, alguna decisión hay que tomar cuando se carece de estudios a largo plazo, por lo cual uno puede tomar la postura conservadora de posponer la decisión hasta que haya datos a largo plazo o tomar una decisión transitoria en base a los resultados de un modelo.

Como las evaluaciones económicas integran en general evidencia de muchas fuentes, es esencial que la misma sea de buena calidad metodológica. En el caso de nuestros estudios, ambos se basan en la mejor evidencia disponible (ensayos clínicos controlados aleatorizados en el caso de la SOMF, y estudios de evaluación de características operativas en el caso de la colonoscopía, sigmoidoscopía y enema de bario).

\section{¿Identifica todos los costos y efectos que debería,y los mide y valora en forma adecuada?}

En este punto uno se debe preguntar si se identificaron tanto los costos como los resultados clínicos que uno considera razonables para esta pregunta, y si las fuentes de donde se extrajeron parecen confiables y precisas.

Ambos estudios miden los costos relacionados a la salud: costos de los tests diagnósticos, sus complicaciones, el costo del cuidado por cáncer colorrectal: la principal diferencia es que el de Frazier toma datos muy detallados de una importante prepaga de EE.UU. y el de Sonnenberg datos menos detallados, pero de un organismo central de todo EE.UU.

Un dato de utilidad para el lector es el reporte separado de las cantidades de recursos utilizados (tests diagnósticos, días de internación, etc.) y por otro lado el valor de cada recurso individual (por ejemplo día de internación, costo de la SOMF). De este modo el lector puede muchas veces comparar su contexto de trabajo y evaluar la aplicabilidad de los resultados. Esto es más importante para nosotros que leemos evaluaciones extranjeras donde muchas veces los costos son muy distintos. Ninguno de nuestros dos estudios evaluados reporta las cantidades y los costos unitarios de manera discriminada.

Existen distintos métodos para la medición de costos, pero básicamente el costeo involucra la estimación del consumo de recursos (por ej.días de internación) y sus costos (costos unitarios).El estudio de Sonnenberg usa los precios publicados por Medicare de EE.UU. Sin embargo los precios pueden diferir de los costos reales dependiendo de la sofisticación del sistema contable y el 
poder de negociación de las instituciones de salud. A veces son necesarios ajustes para pasar los precios a los costos, pero este es un campo muy controvertido y poco estudiado.

Otro dato técnico en el que no abundaremos es que evaluaciones de problemas a largo plazo deben explicar qué descuento realizan a costos y beneficios futuros. Esto tiene que ver con la famosa frase:más vale pájaro en mano (hoy) que cien volando (en un futuro incierto).Ambos estudios utilizan una similar tasa de descuento.

\section{¿Fue el tipo de análisis adecuado a la pregunta realizada?}

Aunque esto suene difícil, no lo es tanto.Vean la tabla 3 e intenten contestarse las siguientes preguntas:

¿Existe una manera más barata (e igualmente efectiva) de cuidar a estos pacientes? En este caso si todas las estrategias son igualmente efectivas, me olvido de la efectividad y solo reporto los costos de cada una, haciendo un análisis de costo-minimización

¿Qué manera de tratar a estos pacientes me va a producir "más salud" por cada peso gastado? En el caso de comparar estrategias para un mismo problema (por ejemplo cáncer colorrectal) la salud se puede medir en años de vida ganados, en número de cánceres prevenidos, etc.El tipo de análisis en este caso es el de costo-efectividad. Es el caso de nuestros dos estudios seleccionados.Ambos reportan sus resultados del mismo modo:en $\mathrm{U} \$ \mathrm{~S}$ por año de vida ganado.

También existe el caso en donde se quieren comparar estrategias para distintas intervenciones o para distintos resultados.Por ejemplo un director de una prepaga debe decidir si cubre una droga nueva para demencia senil o un nuevo anticuerpo monoclonal para la leucemia aguda. En estos casos debo medir con una misma vara los resultados de salud en enfermedades tan disímiles.

La manera más habitual de realizarlo es a través de un análisis de costo-utilidad, en donde los efectos en la salud (tanto buenos como malos) se traducen a "años vividos en perfecta salud". Un ejemplo muy utilizado en la literatura es el uso de QALYs (en inglés años de vida ajustados por calidad). Como ejemplo sencillo, si la sociedad o los pacientes valoran en 0.2 en una escala de 0 (la muerte) a 1 (la salud perfecta) la vida luego de un ACV mayo. Cada año de vida en ese estado se va a contar como 0.2 QALYs, y cinco años en ese estado sumarán 1 QALY. Ninguno de nuestros estudios intenta realizar un análisis de costo-utilidad.

El análisis de costo-beneficio es otra manera menos utilizada hoy en día por muy controvertida, ya que la vara que utiliza para medir los beneficios en la salud (no solo los costos) es el dinero. Como ejemplo, puede preguntar cuánto se estaría dispuesto a pagar para curarse de tal enfermedad, y de esa manera valuar el beneficio de curarla.

\section{¿Se dio cuenta de la incertidumbre en el análisis?}

La incertidumbre en una evaluación económica puede venir de muchos frentes, desde la falta de precisión de estimaciones de efectividades, de costos, de calidad de vida, hasta de controversias metodológicas.

La manera habitual de lidiar con ella es a través del "análisis de sensibilidad", en donde las estimaciones de varios parámetros claves son evaluadas para testear la robustez de las conclusiones del estudio.

Ambos estudios reportan extensos análisis de sensibilidad, in cluyendo la adherencia a los rastreos, frecuencia de realización de los tests, sensibilidad y especificidad de los tests, incidencia de pólipos y costos (el de Frazier incluye también mortalidad de colonoscopía, tasa de recurrencia de pólipos, y progresión de pólipo a cáncer).

En las discusiones de los estudios es otro lugar en donde se reportan en general las incertidumbres metodológicas. Es importante recordar que las evaluaciones económicas son simplemente una ayuda para la toma de decisiones, ya que existen en general muchos difíciles juicios de valores al llegar a una decisión.

En este articulo hemos resumido algunas de las características que debe tener una evaluación económica para ser válida. En el próximo nos dedicaremos a cómo determinar e interpretar los resultados, y a cómo utilizarlos en la práctica.

\section{Dr. Federico Augustovski [ Unidad de Medicina Familiar y Preventiva.Hospital Italiano de Buenos Aires ]}

Nota de último momento: cuando este artículo estaba yendo a imprenta, el médico de familia descubrió, desolado, que había recién salido del horno (científico) una revisión de estudios de costo-efectividad de la US Preventive Srevices Task Force de EE.UU, en la que incluyen los estudios aquí estudiados y más...pero las decisiones hay que tomarlas en tiempo real.Para los interesados, la cita es Pignone et al.Annals of Internal Medicine 2002;137:96-104.

\section{Referencias}

1.O'Brien BJ, Heyland D, Richardson WS, Levine M, Drummond MF. Users' guides to the medical literature. XIII.How to use an article on economic analysis of clinical practice.B. What are the results and will they help me in caring for my patients? Evidence-Based Medicine Working Group.JAMA.1997 Jun 11;277(22):1802-6.

2.Drummond MF, Richardson WS, O'Brien BJ, Levine M. Heyland D. Users' guides to the medical literature. XIII. How to use an article on economic analysis of clinical practice. A.Are the 2.Drummond MF, Richardson WS, O'Brien BJ, Levine M, Heyland D. Users' guides to the medical literature. XII.
results of the study valid? Evidence-Based Medicine Working Group.JAMA.1997 May 21;277(19):1552-7.

results of the study valid? Evidence-Based Medicine Working Group.JAMA.1997 May 21;277(19):1552-7.
3.Detsky AS, Naglie IG.A clinician's guide to cost-effectiveness analysis.Ann Intern Med.1990 Jul 15;113(2):147-54

4.Drummond MF, Jefferson TO.Guidelines for authors and peer reviewers of economic submissions to the BMJ.The BMJ Economic Evaluation Working Party. BMJ.1996 Aug 3;313(7052):275-83 5.M.R.Gold, J.E Siegel, L.B.Russell, and M.C.Weinstein (eds).Cost-effectiveness in Health and Medicine.New York:Oxford University Press, 1996.

6.Drummond MF et al.Methods for economic evaluations of health care programmes.Oxford, England:Oxford University Press;1997.

7.Sacket, Strauss, Richardson, Rosenberg, Haynes.Evidence-bsed medicine.Second Edition.Churchill Livingstone.2000.

8.Frazier AL, Colditz GA, Fuchs CS et al.Cost-effectiveness of screening for colorectal cancer in the general population.JAMA 2000;284:1954-61

9.Sonnenberg A, Delco F y Inadomi JM.Cost-effectiveness of colonoscopy in screening for or colorectal cancer.Ann Intern Med 2000;133:573-84 